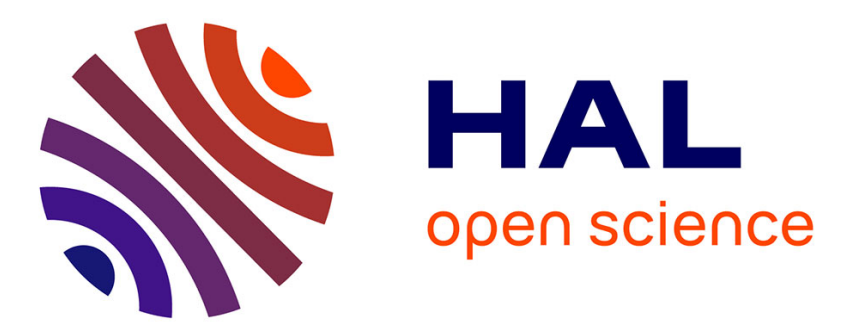

\title{
Test of a recoil ion source at GANIL medium energy facility
}

A. Gosselin, P. Boduch, D. Hennecart, S. Hicham, X. Husson, D. Lecler, A. Lepoutre, A. Cassimi, J. Grandin

\section{To cite this version:}

A. Gosselin, P. Boduch, D. Hennecart, S. Hicham, X. Husson, et al.. Test of a recoil ion source at GANIL medium energy facility. Journal de Physique III, 1994, 4 (9), pp.1765-1777. 10.1051/jp3:1994239 . jpa-00249223

\section{HAL Id: jpa-00249223 https://hal.science/jpa-00249223}

Submitted on 1 Jan 1994

HAL is a multi-disciplinary open access archive for the deposit and dissemination of scientific research documents, whether they are published or not. The documents may come from teaching and research institutions in France or abroad, or from public or private research centers.
L'archive ouverte pluridisciplinaire HAL, est destinée au dépôt et à la diffusion de documents scientifiques de niveau recherche, publiés ou non, émanant des établissements d'enseignement et de recherche français ou étrangers, des laboratoires publics ou privés. 
Classification

Physics Abstracts

$34.50 \mathrm{H}-34.70-41.80 \mathrm{D}$

\title{
Test of a recoil ion source at GANIL medium energy facility
}

\author{
A. Gosselin ( $\left.{ }^{1}\right)$, P. Boduch ('), D. Hennecart ('). S. Hicham ('), X. Husson ('), \\ D. Lecler $\left({ }^{1}\right)$, A. Lepoutre $\left({ }^{1}\right)$, A. Cassimi $\left({ }^{2}\right)$ and J. P. Grandin $\left({ }^{2}\right)$ \\ (') Laboratoire de Spectroscopie Atomique associé au CNRS n 19, ISMRA et Université de \\ Caen, 14050 Caen cedex. France \\ (2) CIRIL, Laboratoire mixte CEA CNRS, B.P. 5133, 14040 Caen cedex. France
}

(Received 17 February 1993, revised 5 Aprll 1994, accepted 30 Mav 1994)

\begin{abstract}
Résumé. - Une source d'ıons de recul, destinée à la réalısation d'expériences de collisions d'ions multichargés de très basse énergie, a été mise au point et testée sur la SME (Sortie à Moyenne Energie) du GANIL (Grand Accélérateur National d'Ions Lourds). L'efficacité des faisceaux de la SME pour la production d'ions de recul d'argon de hauts états de charge est mise en évidence (plus de $10^{4}$ ions $\mathrm{Ar}^{15+}$ obtenus par seconde pour un faisceau d'ions projectiles de $300 \mathrm{nA}$ d'ions $\mathrm{Xe}^{39+}$ de $4,7 \mathrm{MeV} / \mathrm{A}$ ). Il est de plus démontré que les faisceaux produits par cette source peuvent être décélérés jusqu'à des énergies de l'ordre de quelques qeV sans perte importante d'intensité ni de qualité.
\end{abstract}

\begin{abstract}
A recoil ion source, devoted to the production of very low energy multicharged ion beams which are of interest for charge exchange experiments, has been designed and tested at the SME (medium energy facility) of GANIL (heavy ion accelerator in Caen, France). The efficiency of SME beams for the production of high charge-states of argon recoil ions is demonstrated (more than $10^{+} \mathrm{Ar}^{15+}$ ions/s obtained with a $300 \mathrm{nA} 4.7 \mathrm{MeV} / \mathrm{A} \mathrm{Xe} \mathrm{Xe}^{39+}$ projectile ion beam). It is shown furthermore that these beams may be decelerated down to energies of some qeV without dramatic loss of intensity and quality.
\end{abstract}

\section{Introduction.}

Experimental results about charge exchange collisions at very low energy between multicharged ions and neutral targets deserve interest, not only from a fundamental point of view, but also for possible applications in the domains of astrophysical and fusion plasmas $[1,2]$. Such results are relatively scarce. owing to the fact that production and handling of very low energy multicharged ion beams still constitute an experimental challenge. Concurrently to the method which consists in slowing down ion beams extracted from conventional (ECR or EBIS) electron impact ion sources. recoil ion sources (RIS) have been operated at several heavy ion 
accelerators. RIS make use of a swift multicharged heavy ion beam to ionize the atoms or molecules of a gas-target and have been demonstrated to be efficient devices for the production of low energy multicharged ion beams with reasonable energy dispersion [3-7]. A swift multicharged heavy ion reveals indeed to be very efficient for stripping, in a single collision, a large number of electrons from a neutral target atom while imparting only a very small quantity of energy to its nucleus.

The ionization efficiency of accelerated heavy ions lies in the combined effects of their high charge-states and high velocities. The former insures efficient ionization of outer electrons of the target atom while the latter provides the possibility of inner shell ionizations further completed by Auger decay of the resulting excited ion. Low energy accelerators, such as Van de Graaf tandems which deliver ion beams in the MeV/A energy range, have been used successfully for the production of high charge-states of light elements like neon [4]. These accelerators revealed however deceiving for the production of highly charged recoil ions of argon. The velocities of their heavy ion beams are indeed too low to permit efficient hole creation in argon $\mathrm{K}$-shell. Higher energy accelerators have of course been used and have proved, in principle, their efficiency for the production of high charge-state recoil ions for heavier elements. These have been Unilac at Darmstadt [8], Super Hilac at Berkeley [9] or GANIL at Caen [10], promising estimations have also been done concerning SIS-ESR at Darmstadt [11]. Nevertheless, an experimental program for the study of low energy collisions appears to be rather time consuming and hardly compatible with the saturated time schedule of such machines.

At GANIL however, the medium energy facility (SME) provides since 1990 intense beams ranging from $\approx 14 \mathrm{MeV} / \mathrm{A}$ argon to $\approx=4 \mathrm{MeV} / \mathrm{A}$ uranium ions. These beams are fully dedicated to atomic and solid-state physics and seem furthermore to be very well suited for the stripping of argon atoms. These are the reasons which have motivated our interest for the design of a new RIS adapted to this facility.

We present in this paper a description of the source and the results of tests which were performed at SME. These tests were mainly devoted to the evaluation of available intensities, energy dispersions and optical qualities of the RIS beams. The conditions upon which such beams may be slowed down are analysed in detail.

\section{Experimental set-up.}

The experimental set-up used (Fig. 1) consists in the RIS itself, completed by a set of detectors which allow the characterization of the extracted beam. The whole set-up, source plus detectors, is placed in a vacuum chamber which can be connected either to the GANIL beam line, or to an electron gun for « off line » test experiments.

1.1 The ReCOIL ION SOURCE. - Production of the recoil ions is performed by sending the projectile beam (parallel to the $\mathrm{O} y$ direction of Fig. 1) through a gas cell from which a weak electric field (parallel to the $\mathrm{O} z$ direction) extracts the recoil ion beam. This beam is then accelerated and focused in order to allow a good charge-state separation by the charge analysis device. The extracting and accelerating electric fields communicate to ions of charge $q$ and mass $m$, velocities which are proportional to the square root of their $q / m$ ratio. Charge-state analysis is then performed by a Wien-filter (WF) which is a velocity selective device. The charge-state selected ion beam may at last be decelerated to the desired energy value by mean of a retarding electric field.

1.1.1 Production and extraction. - The production of recoil ions takes place in a flat cylindrical differentially pumped gas cell. Two opposite $5 \mathrm{~mm}$ diameter holes permit the 

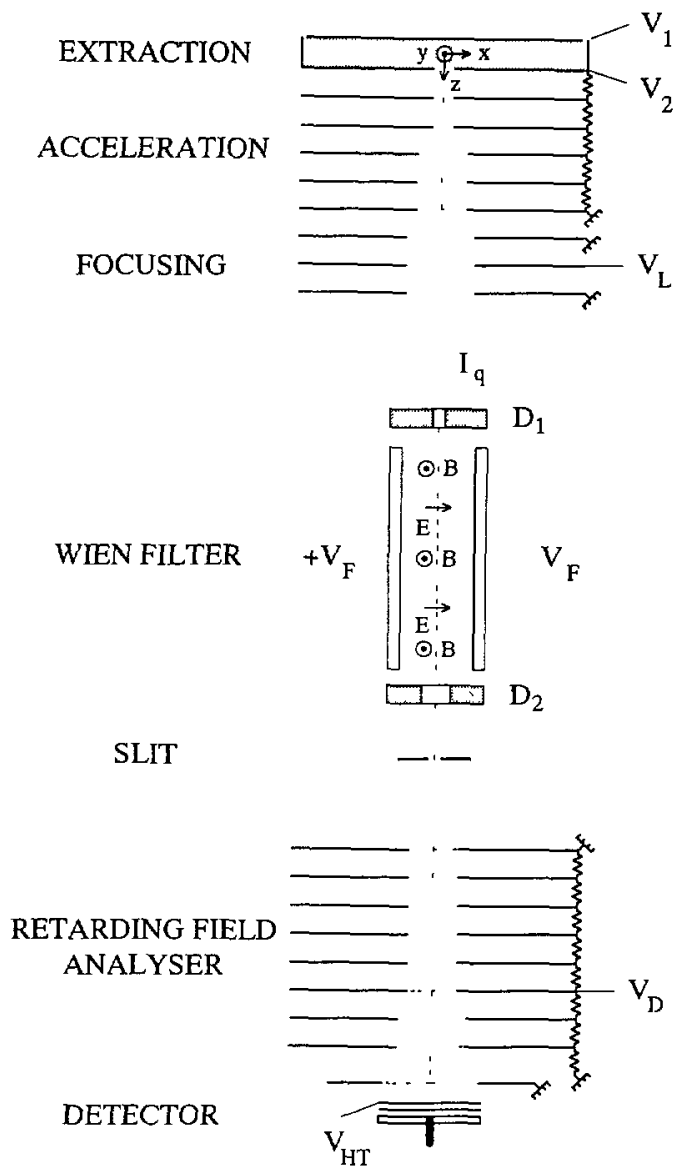

Fig. 1. - Scheme of the experimental set-up.

passage of the projectile ion beam along a diameter of the cell, gas feeding and pressure measurements are performed through two different pipes also connected to the cell. The two insulated end caps of the cell, distant of $9 \mathrm{~mm}$, constitute electrodes which, respectively biased to potentials $V_{1}$ and $V_{2}$ (with $V_{1}>V_{2}$ ), allow to apply the extracting electric field (a few $\mathrm{V} / \mathrm{cm}$ ). Extraction thus occurs, perpendicularly to the projectile beam direction, at the centre of the cap at potential $V_{2}$, through a $3 \mathrm{~mm}$ diameter hole. The quantity $V_{1}-V_{2}$ will hereafter be referred to as " extraction voltage ", while the quantity $V_{0}=\left(V_{1}+V_{2}\right) / 2$ will be referred to as «mean acceleration voltage ».

1.1.2 Acceleration and focusing. - Beam acceleration is ensured by a set of five $90 \mathrm{~mm}$ diameter $1 \mathrm{~mm}$ thick plates. These plates are connected by a chain of resistors which allow to uniformly divide the accelerating voltage $V_{2}$ over the acceleration region. Three additional plates, among which the two extremes are grounded while the middle one is biased to a potential $V_{\mathrm{L}}$, constitute a focusing Einzel-lens. In order to minimize fringing effects, the holes opened through the plates for the passage of the ion beam are covered with $90 \%$ transparency nickel meshes, at each place in the experimental set-up where their position along the beam axis corresponds with a gradient of the electric field (except of course for the Einzel-lens). 
1.1.3 Charge-state selection. - The ion beam is submitted in the WF to both an electric field $\mathbf{E}$ and a magnetic field $\mathbf{B}$ (respectively parallel to the $O x$ and $O y$ directions). An ion of velocity $\mathrm{v}$ and charge $q$ will then feel, along the electric field direction, a force $: q(E-v B)$ which vanishes for a given velocity, provided that proper values of $E$ and $B$ are chosen. In the present case, the magnetic field is fixed and created by two $97 \mathrm{~mm}$ long, $30 \mathrm{~mm}$ wide permanent magnets which allow to reach a mean field value of $0.1 \mathrm{~T}$ across a $20 \mathrm{~mm}$ gap. The electric field is created by two $97 \mathrm{~mm}$ long $18 \mathrm{~mm}$ wide plates, distant of $30 \mathrm{~mm}$ and symetrically biased to $\pm V_{\mathrm{F}}$ in order to keep the axis of the filter at zero potential. The active length of the WF is limited by two soft iron rings $D_{1}$ and $D_{2}$. A slit ( $2 \mathrm{~mm}$ width), parallel to the magnetic field, placed at $30 \mathrm{~mm}$ behind the output of the WF, allows to select the desired charge state.

1.1.4 Final energy adjustment. - Final energy adjustment consists in decelerating the charge-state selected beam. This is done over a distance of $50 \mathrm{~mm}$ by the mean of a retarding electric field, this field is produced by six plates which are connected by a chain of resistors allowing to uniformly divide the decelerating voltage $V_{\mathrm{D}}$.

1.2 The Detectors. - Two detectors are used in order to determine the intensity, the size and the divergence of the produced ion beam.

- A Faraday cup, equipped with a biased guard-ring, can be inserted between the output of the WF and the entrance of the decelerating stage, it allows to measure the absolute intensity of the charge-state selected beam before deceleration.

- A position sensitive detector (PSD) [12], made of a set of two microchannel plates (MCP) followed by a resistive anode, allows both to count the ions behind the deceleration stage and to localize their impact with a resolution of the order of $100 \mu \mathrm{m}$ providing thus the intensity and the shape of the beam. As the distance between this PSD and the output of the ion source may be varied, information can furthermore be obtained on the divergence of the beam.

The ions have of course to be re-accelerated in order to ensure a good efficiency of the MCP detector, two additional plates are used for that purpose.

$V_{\mathrm{F}}$ or $V_{\mathrm{D}}$ voltages may at last be driven by a microcomputer which simultaneously allows to record the counts of the MCP detector.

\section{Performances of the RIS.}

Accelerator experiments have been preceded by numerical simulations and experimental tests using electrons issued from a $2 \mathrm{keV}$ electron gun as ionizing projectiles. They enabled us to precisely define the ranges of acceleration $V_{2}$ and lens $V_{\mathrm{L}}$ voltage values through which proper charge-state selection and transport conditions of the RIS beam may be achieved.

Figure 2 demonstrates the effectiveness of the extraction and charge-state selection devices for high charge-states of argon recoils, as well as the ionization efficiency of a $4.7 \mathrm{MeV} / \mathrm{A}$ $\mathrm{Xe}^{39+}$ projectile beam.

Tests presented hereafter were performed at GANIL/SME, they have consisted in studying the influence on the intensities, shapes and energy dispersions of the obtained recoil ion beams, of various parameters which are : the pressure of the gas in the production cell, the extraction voltage $V_{1}-V_{2}$ and the deceleration voltage $V_{\mathrm{D}}$.

\subsection{BEAM INTENSITY.}

2.1.1 Influence of gas target pressure. - With increasing gas pressure in the target cell, the probability for producing a recoil ion with a given charge-state is expected to increase, as well as, due to electron capture, the probability for such an ion to have its charge state lowered before being extracted. 


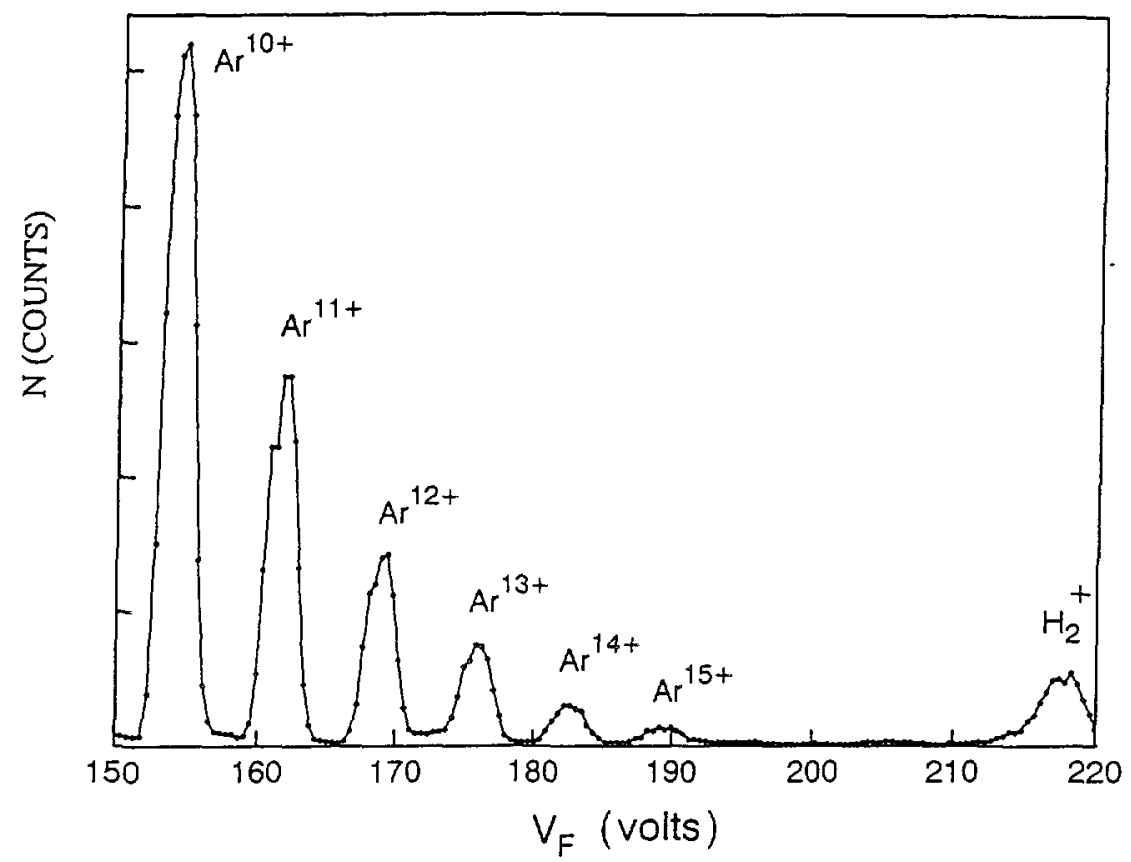

Fig. 2. - Number $\mathrm{N}$ of MCP detected ions versus $\mathrm{Wien}$-filter voltage $V_{\mathrm{F}}$ (projectile beam : $4.7 \mathrm{MeV} / \mathrm{A}$ $\mathrm{Xe}^{79+} V_{1}-V_{2}=2 \mathrm{~V}, V_{2}=449 \mathrm{~V}, V_{L}=465 \mathrm{~V}, V_{\mathrm{D}}=0$ ).

A very simple model allows to write the number $N_{4}$ of extracted ions of charge $q$, if one neglects the re-population of this charge-state by electron capture from higher charge states :

$$
N_{q}=N_{\mathrm{p}} \cdot \sigma_{q}^{1} \cdot n \cdot \ell_{1} \cdot\left(1-n \cdot \sigma_{q}^{c} \cdot \ell_{2}\right)
$$

where $N_{\mathrm{p}}$ is the number of projectile ions, $\sigma_{q}^{\prime}$ and $\sigma_{q}^{\mathrm{c}}$ are respectively the total effective cross sections: for the production of a recoil ion of charge $q$ and for electronic capture by such a recoil ion from a neutral argon target, $\ell_{1}$ and $\ell_{2}$ are respectively: the useful length of the gas cell along the projectile beam axis and the mean distance along which the recoil ions travel before leaving the cell, and $n$ is the number of target atoms per unit volume in the gas cell. Such a relation describes a parabolic behaviour of $N_{q}$ as a function of $n$ with a maximum occurring for $\frac{1}{2 \cdot \sigma_{q}^{c} \cdot P_{2}}$ It allows at least to expect the existence of a finite optimum pressure for the production of each beam with a given charge-state.

Figure 3 displays the variations of extracted argon beam intensities versus gas pressure into the cell. These intensities have been determined with the Faraday cup for charges $q=1$ to 8 and with the MCP detector for charges $q=4$ to 15 . Calibration of MCP measurements with regard to Faraday cup measurements has been achieved using the results for charges $q=4$ to 8 which could be measured with both detectors. Operation of the MCP detector is indeed limited to a maximum pressure in the cell of $4 \times 10^{-3}$ Torr, beyond which the residual pressure in the experimental chamber $\left(>2 \times 10^{-5}\right.$ Torr $)$ gets too high to allow the MCP detector to be switched on. For charge-states $q>2$, the existence of optimum pressures for recoil ion production is clearly visible. One can notice that, in the frame of this model and in spite of its roughness : 


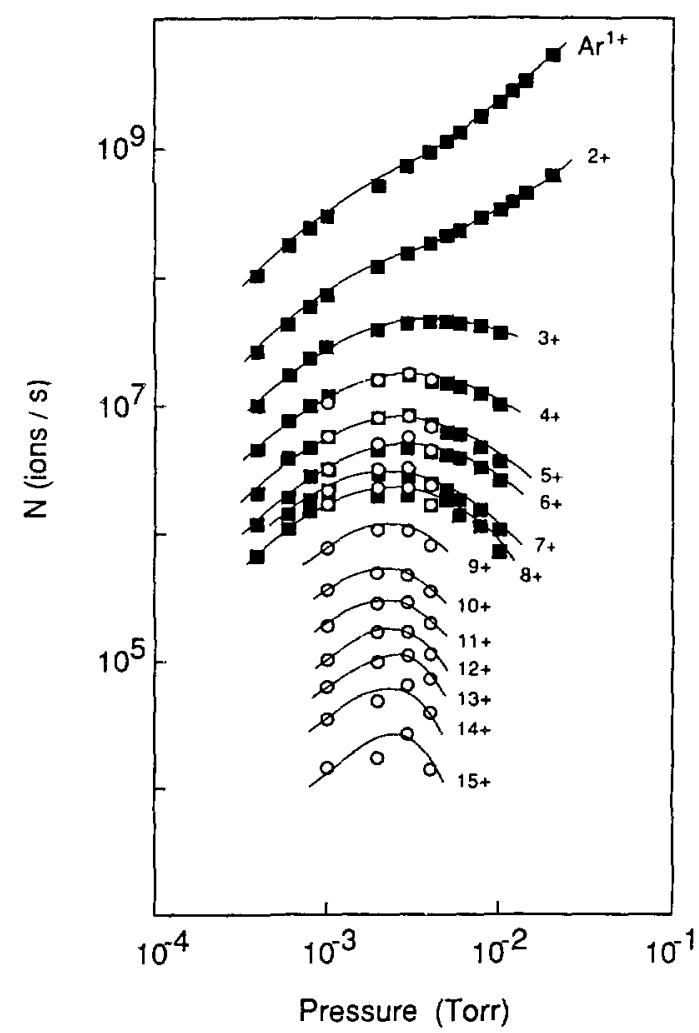

Fig. 3. - Beam intensities $N$ (ions/s) versus gas pressure into the target cell (projectile beam : $300 \mathrm{nA}$, 4.7 MeV/A Xe ${ }^{39+}$ ). Full squares: measurements performed with the Faraday cup, Open circles: measurements performed with the MCP detector.

- the positions of the observed maxima ( $)$ (in the $10^{-3}-10^{-2}$ Torr range) are compatible with realistic values of $\ell_{2}$ (in the $\mathrm{cm}$ range) and $\sigma_{q}^{\mathrm{c}}$ (in the $10^{-14}-10^{-15} \mathrm{~cm}^{2}$ range);

- the shift of the observed maxima towards lower pressures for increasing $q$ values qualitatively reflects, at least up to $q=8$, the well known increase with $q$ of $\sigma_{q}^{c}$ values.

Figure 3 also allows to emphasize on the efficiency of the $4.7 \mathrm{MeV} / \mathrm{A} \mathrm{Xe} \mathrm{e}^{39+}$ beam, which provides more than $10^{4} \mathrm{Ar}^{15+}$ ions per second, by comparing it to the efficiency of the $30 \mathrm{MeV} \mathrm{Cl}^{5+}$ beam used by the authors of reference [7], who quote $5 \times 10^{3} \mathrm{Ar}^{6+}$ per second.

Captures which may occur from the residual gas along the transport path, will have different consequences according to whether they occur in the equipotential region, whether they occur during the extraction-acceleration stage. The ions for which a capture occurs in the equipotential region will constitute a component of lower charge-state in a beam of selected charge-state $q$, it will be shown further (see Sect. 2.3) that their number remains, in the percent range of the total number of ions in the beam. Ions having captured an electron during the acceleration stage will have different energies depending on the exact place at which the capture occurred, these ions will then be spread over the whole energy spectrum and contribute to a background appearing on spectra similar to the one displayed in figure 2 . Such

(1) It is worth in practice to notice that the observed maxima occur for pressures which are fully compatible with satisfying beam transport and MCP operatıng conditions. 
backgrounds have always been negligible, whatever the pressure used in the domain which has been explored, probably due to a fast drop of gas pressure along the recoil ion beam path after the output of the cell. At last, ions of charge $q+1$ having captured an electron in the extraction region, very close to their production place, will have a charge $q$ and an energy similar to ions directly produced with charge $q$, except for a slight difference corresponding to the energy gained during the charge exchange collision. Further experiments which will be described elsewhere, have allowed us to observe such ions in a ratio of only a few per cent of the main beam. Such a number of ions is actually to small to significatively perturb the energy dispersion measurements described in section 2.3. It remains however important to keep in mind the existence of such parasitic beams (having the same charge but not exactly the same energy or the same energy but not the same charge as the main beam) even of low intensity. since they can hinder future collision experiments making use of these beams except if convenient experimental procedures are used.

2.1.2 Influence of extraction voltage. - The recoil ion beam intensity has been determined using the Faraday cup, for fixed gas pressure and incident ion flux, as a function of extraction voltage $V_{1}-V_{2}$. As shown in figure 4 the extraction efficiency, which drops of course for the lowest extraction voltages, remains otherwise almost independent of extraction voltage. Such a behaviour evidences the fact that for a sufficient extraction voltage all the produced ions are extracted from the cell. The slight maxima observed around $V_{1}-V_{2}=5 \mathrm{~V}$ have already been observed by the authors of reference [7] and attributed by them to residual lens effects occurring, in spite of the presence of a grid at this place, at the passage through the extraction hole of the production cell.

Variations entirely similar to the one displayed in figure 4 have been observed for different gas pressures in the range $10^{-1} 3 \times 10^{-2}$ Torr.

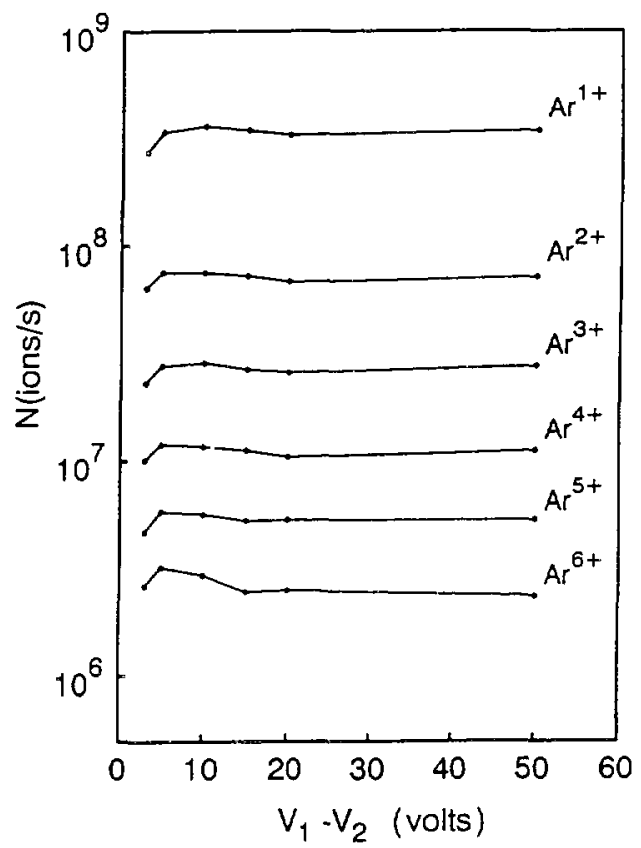

Fig. 4. - Beam intensities $N$ (ions/s) versus extraction voltage $V_{1}-V_{2}$ (projectile beam: $300 \mathrm{nA}$, 4.7 MeV/A Xe $\mathrm{X}^{39+}$ gas pressure into the cell $\left.10^{-7} \mathrm{Torr}\right)$. 
2.2 Divergence, emittance And brightness of the beam. - The optical characteristics, divergence, emittance and brightness, of the charge state analysed beam will be considered in this paragraph. They are the one in which we are interested for further experiments making use of this beam and may be estimated using the previously described PSD.

Due in particular to the astigmatic focusing properties of the Wien filter [13], it is actually not possible to obtain a recoil ion beam focused exactly at the same point along $z$ axis in directions parallel to $\mathrm{O} x$ an $\mathrm{O} y$. Already mentioned preliminary tests, as well as verifications made during the experiment, have shown however that it was possible to find a value of the lens potential for which every recoil ion beam with charge state higher than $q=3$ could be considered as reasonably focused in both directions somewhere between the output of the WF and the point of closest approach of the detector which is situated at $135 \mathrm{~mm}$ from this output. With a mean extraction voltage $V_{0}=450 \mathrm{~V}$ these conditions are for instance obtained for a $V_{\mathrm{L}}$ value of about $470 \mathrm{~V}$.

The observed characteristic dimensions $\Delta x$ and $\Delta y$ (fwhm) of recoil ion beams of charge states $q=5,10$ and 14, determined at distances of 135,235 and $335 \mathrm{~mm}$ from the output of the Wien filter, are given in table I. Also given in this table are the divergences $\Delta \theta$, and $\Delta \theta$, which may be deduced from these values.

Considering $\Delta x$ and $\Delta y$ values obtained at $135 \mathrm{~mm}$ from the output of the WF as the size of the focused beam $\left({ }^{2}\right)$ and using the determined divergences values, one can deduce upper limits for the normalized transverse emittances defined as :

$$
\varepsilon_{1}=\pi \cdot \Delta x \cdot \Delta \theta_{1} . V / c \text { and } \varepsilon_{1}=\pi \cdot \Delta y \cdot \Delta \theta_{1} . V / c
$$

where $V$ is the longitudinal velocity of the recoil ion beam and $c$ the speed of light [1]. The corresponding values are given in table I.

Table I gives at last, according to the characteristic current $I$ (particle-pA) obtained for each charge state (see for instance Fig. 3), lower limits for the emittance normalized brightness :

$$
\mathrm{Br}=I / \varepsilon_{1} \cdot \varepsilon_{1}
$$

Table I. - Dimensions $\Delta$, divergences $\Delta \theta$, emittances $\varepsilon$ and brillances $\mathrm{Br}$ of $\mathrm{Ar} 5^{+}, 10^{+}$and $14^{+}$beams (projectile beam : $4.7 \mathrm{MeV} / \mathrm{A} X \mathrm{Xe}^{39+}, V_{1}-V_{2}=2 \mathrm{~V}, V_{2}=449 \mathrm{~V}, V_{\mathrm{L}}=465 \mathrm{~V}$, $V_{\mathrm{D}}=0$ ).

\begin{tabular}{|c|c|c|c|}
\hline Charge-state $q$ & $5^{+}$ & $10^{+}$ & $14^{+}$ \\
\hline$\Delta_{x} / \Delta_{y}(135 \mathrm{~mm}): \mathrm{fwhm} \mathrm{mm}$ & $\pm 0.9 / \pm 2.1$ & $\pm 0.7 / \pm 1.7$ & $\pm 0.6 / \pm 1.9$ \\
\hline$\Delta_{x} / \Delta_{y}(235 \mathrm{~mm}): \mathrm{fwhm} \mathrm{mm}$ & $\pm 1.3 / \pm 2.9$ & $\pm 1.4 / \pm 2.5$ & $\pm 1.8 / \pm 3$ \\
\hline$\Delta_{x} / \Delta_{y}(335 \mathrm{~mm}): \mathrm{fwhm} \mathrm{mm}$ & $\pm 1.7 / \pm 3.6$ & $\pm 2.1 / \pm 3.2$ & $\pm 3 / \pm 3.9$ \\
\hline$\Delta \theta_{x} / \Delta \theta_{v}: \mathrm{mrad}$ & $\pm 4 / \pm 7.5$ & $\pm 7 / \pm 7.5$ & $\pm 12 / \pm 10$ \\
\hline$\varepsilon_{x} / \varepsilon_{y}: \pi \mathrm{mm} \mathrm{mrad}$ & $1.25 \times 10^{-3} / 5 \times 10^{-3}$ & $2.5 \times 10^{-3} / 6 \times 10^{-3}$ & $4.5 \times 10^{-3} / 10^{-2}$ \\
\hline $\mathrm{Br}:$ part. $\mathrm{pA} /(\pi \mathrm{mm} \mathrm{mrad})^{2}$ & $1.6 \times 10^{4}$ & $4.8 \times 10^{2}$ & 16 \\
\hline
\end{tabular}

(2) These values are actually in excess since the different beams are more or less focused in front of this position. 
Divergences are observed to increase with charge state, $\Delta \theta_{1}$ increasing even more rapidly than $\Delta \theta_{v}$. This is not surprising, the optical characteristics of the beam are indeed expected to evolve with charge-state in different ways along directions parallel to $O x$ and $O y$ due to various phenomena such as the anisotropy of recoil velocities (large recoil velocity component in the $x \mathrm{O} z$ plan) $[1,14]$ and the chromaticity of the transport beam line.

2.3 ENERGY DISPERSION, SLOWING DOWN OF THE BEAM. - Slowing down and determining the energy dispersion of the beam are of course tightly bound problems : energy dispersion limits indeed on the one hand the possibility of slowing down the beam, the slowing down procedure provides us on the other hand with a way of determining the energy dispersion.

2.3.1 Intensity of the decelerated beam. - In order to analyse the energy dispersion of the beam, the retarding field method has been used. The principle of the method consists in detecting, for a selected charge-state $q$, the number of ions reaching the MCP detector as a function of increasing values of the deceleration voltage $V_{\mathrm{D}}$.

As long as $V_{\mathrm{D}}$ remains smaller than $V_{0}$, the intensity of the decelerated beam does not vary significantly (Fig. 5). For $V_{\mathrm{D}}=V_{0}$, the number of counts suddenly decreases $\left({ }^{3}\right)$. This decrease corresponds to the repulsion towards the WF of the ions of charge-state q. For values of $V_{\mathrm{D}}>V_{0}$ the recorded signal (less than $2 \%$ of the initial one in the particular case of Fig. 5) corresponds to the MCP background plus the signal due to ions having decreased their charge state by electron capture during the transport from the end of the acceleration stage.

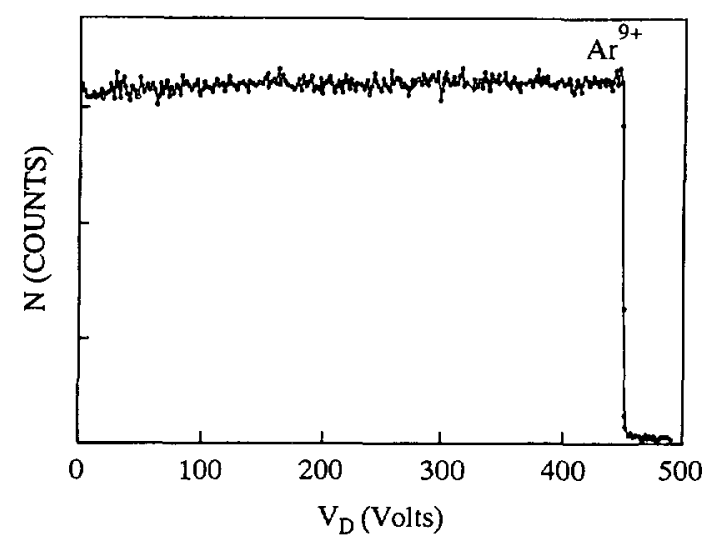

Fig. 5. - Number of $\mathrm{Ar}^{5+}$ recoil ions detected on the MCP detector versus deceleration voltage $V_{\mathrm{D}}\left(V_{1}-V_{2}=2 \mathrm{~V}, V_{2}=449 \mathrm{~V}\right)$.

One observes actually (as it can be seen even more clearly in figure 8 ) that the decrease of the beam intensity around $V_{\mathrm{D}}=V_{0}$ has a certain width $\Delta V_{\mathrm{D}}$ from which the energy dispersion $q . \Delta V_{\mathrm{D}}$ of the beam can be obtained.

The three components of the observed recoil ion beam energy dispersions are expected to be :

- the energy dispersion introduced by the difference of extraction voltage across the width

( ${ }^{3}$ It is important to notice the fact that the $V_{D}$ value for which half of the ions are repelled occurs precisely for $V_{\mathrm{D}}=V_{0}$ (Fig. 5 and Fig. 8 ) since it attests for the good control which can be kept over the beam energy even for the highest deceleration values. 
of the primary beam $\Delta E_{\text {ext }}$ (with a primary beam width of $3 \mathrm{~mm}$ and an extraction field of $3 \mathrm{~V} / \mathrm{cm}$ for instance, an energy dispersion of $\approx 0.9 \mathrm{qeV}$ is expected);

- the instrumental dispersion $\Delta E_{\mathrm{I}}$ including the effect of imperfect transport optic conditions and the limited resolution of our energy analysis method;

- the initial energy dispersion of the recoil ions $\Delta E_{\mathrm{R}}$ which, if one neglects the thermal energy of the target atoms, is due to the recoil energy imparted to the ions during the collision in which they are created and increases with increasing recoil ion charge-state $[14,15]$.

Experimental values of $\Delta V_{\mathrm{D}}$ are given in figure 6 for charge-states $q=1,10$ and 14 of argon, versus the extraction voltage $V_{1}-V_{2} . \Delta V_{\mathrm{D}}$ presents, for each charge-state, a linear variation which may obviously be attributed to the proportionality of $\Delta E_{\text {ext }}$ and $V_{1}-V_{2}$. The observed slope, proportional to the diameter of the projectile beam used, depends on $q$ state. Adjustment of the counting rate of the detector is indeed performed by « cutting » into the projectile beam emittance, smaller (resp. higher) beam diameters and thus slopes then correspond to charge-states which are more (resp. less) abundant.

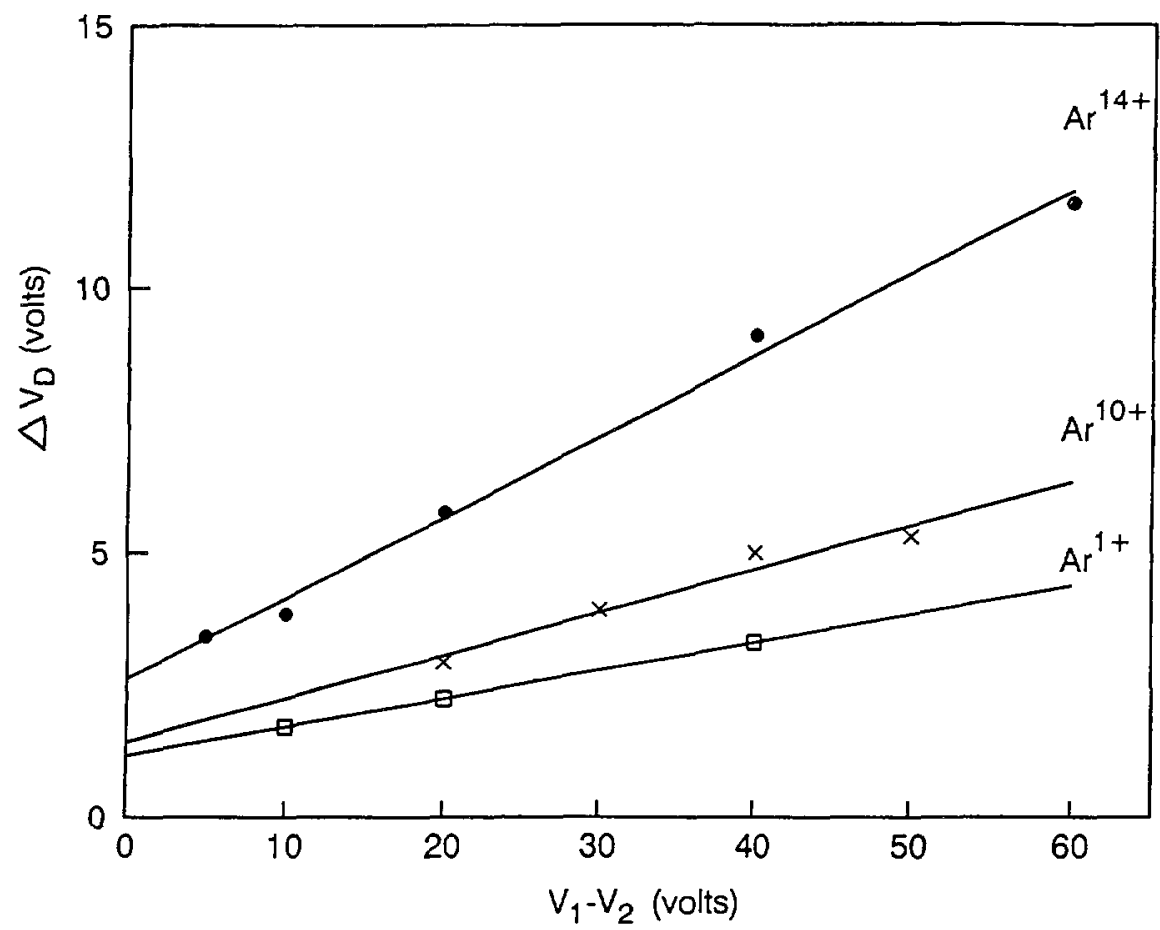

Fig. 6. - Evolution of the width $\Delta V_{\mathrm{D}}$ of the deceleration curve versus the extraction voltage $l_{1}-l_{2}$, for various charge-states.

For each charge-state, extrapolation of $\Delta V_{\mathrm{D}}$ to $V_{1}-V_{2}=0$ leads to the determination of $\Delta E_{\mathrm{I}}+\Delta E_{\mathrm{R}}$. Previous experimental results [14] have shown that in the particular case of $\mathrm{Ar}^{1+}, \Delta E_{\mathrm{R}}$ may be considered as negligible. Extrapolation of $\Delta V_{\mathrm{D}}$ to $V_{1}-V_{2}=0$ for $\mathrm{Ar}^{1+}$ then provides an estimation of $1.6 \mathrm{qeV}$ for $\Delta E_{1}$. If we assume this determination to be valid for any charge-state, we obtain $\Delta E_{\mathrm{R}}$ estimations of respectively 0.2 and $1.6 \mathrm{qeV}$ for charges $q=10$ and 14 .

It does not exist, at our knowledge, experimental determinations of recoil energies which are 
directly comparable to these values. However, using on the one hand the recoil energies obtained by Grandin et al. [14] with a projectile beam of $27 \mathrm{MeV} / \mathrm{A} \mathrm{Xe}^{52+}$ ions, using on the other hand the $Z_{\mathrm{P}}^{2} / E_{\mathrm{p}}$ variation law mentioned by Schlachter [16] for the dependence of recoil energies on projectile charge $Z_{\mathrm{p}}$ and energy $E_{\mathrm{p}}$, one deduces values of 0.1 and $0.9 \mathrm{qeV}$ for the mean recoil energies of charge-state 10 and 14 . These values are perfectly consistent with the $\Delta E_{\mathrm{R}}$ values obtained in the present experiment if we consider $\Delta E_{\mathrm{R}}$ to be equivalent to twice the mean recoil energy.

2.3.2 Shape of the decelerated beam. - It is worth at this point to wonder about the meaning of the pattern observed on the position sensitive detector for such a decelerated re-accelerated beam (Fig. 1). As in fact the re-acceleration occurs on a distance which represents only $2 / 5$ of the length of the deceleration region, the re-acceleration time represents less than $15 \%$ of the total time which separates the beginning of the deceleration from the detection time. The pattern observed on the PSD may consequently be considered as a close image of the beam at its point of lowest velocity.

Such patterns, obtained for an $\mathrm{Ar}^{15+}$ beam with a mean acceleration voltage $V_{0}=450 \mathrm{~V}$ $\left(V_{1}=451 \mathrm{~V}, V_{2}=449 \mathrm{~V}\right)$ and six different values of $V_{\mathrm{D}}$ ranging from 0 to $455 \mathrm{~V}$, are displayed in figure 7 .

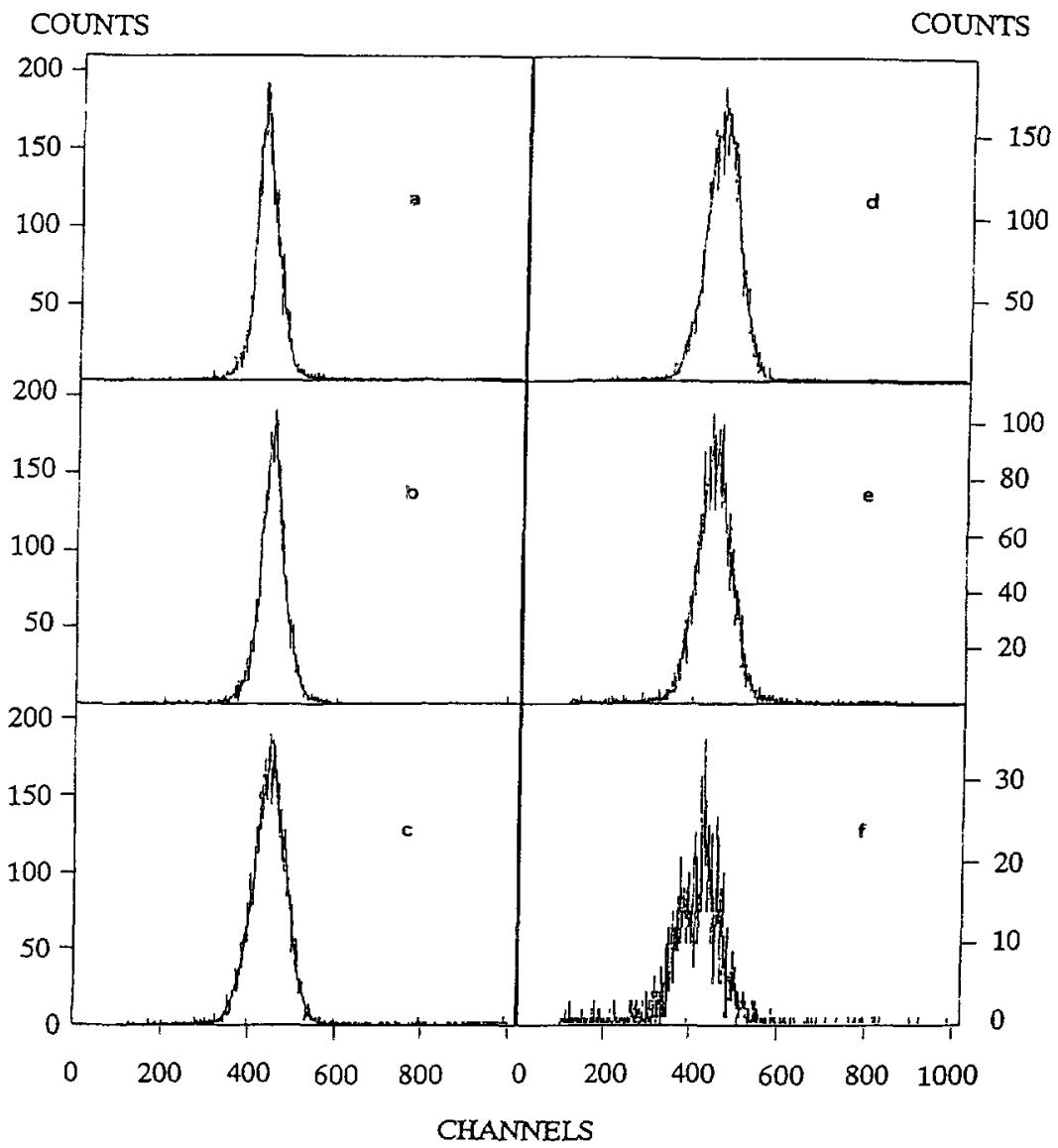

Fig. 7. - Experimental profiles (Ox direction) of a recoll ion beam, the selected charge-state is $15^{+}$, the mean acceleration voltage $V_{0}=450 \mathrm{~V}$, the deceleration voltage $V_{D}=0 \mathrm{~V}$ (a), $440 \mathrm{~V}$ (b), $449 \mathrm{~V}$ (c), $449.5 \mathrm{~V}(\mathrm{~d}), 450 \mathrm{~V}(\mathrm{e}), 455 \mathrm{~V}(\mathrm{f})$. 
Figure 8 shows the deceleration curve obtained for the same beams as explained previously. From this deceleration curve a $\Delta V_{\mathrm{D}}$ value of $4.5 \mathrm{~V}$ is deduced.

The following remarks arise from the simultaneous observation of the patterns of figure 7 and of the corresponding beam intensities in figure 8 :

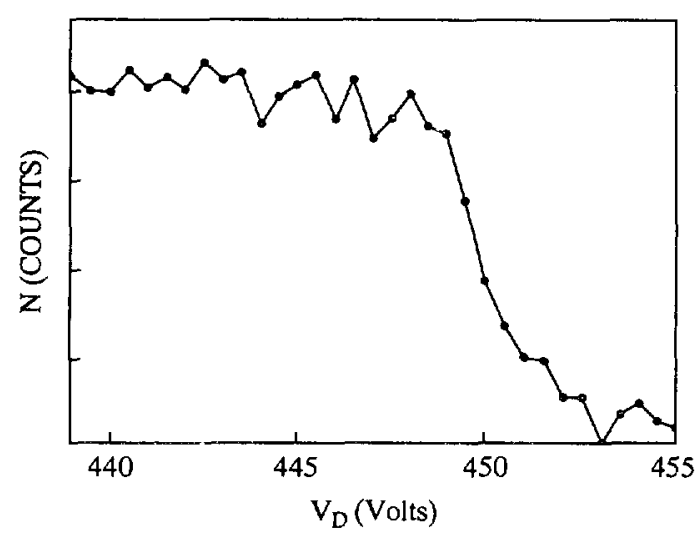

F1g. 8. - Number of recoil ions detected versus deceleration voltage $V_{D}$, same beam as for figure 7 .

- the beam observed in figure $7 \mathrm{f}$ no longer corresponds of course to $\mathrm{Ar}^{15+}$ ions but to ions having changed their charge-state by electron capture; the total number of these ions, once deduced the background count of the MCP, amounts to about $10 \%$ of the selected beam ;

- down to energies of about $10 \mathrm{geV}$ the recoil ion beam may be slowed down without appreciable loss of intentisy and deterioration of shape figures $7 \mathrm{a}, \mathrm{b}$;

- for a more severe slowing down, the observed loss of intensity allows to limit the broadening of the beam as observed from figures $7 \mathrm{c}, \mathrm{d}$, e.

The observation of a correct shape of the beam, even for the lowest energies, may be attributed to the particular velocity distribution in the beam which is a consequence of the initial non thermal recoil ion velocity distribution : the last ions to be rejected in the course of the slowing down are indeed those which have the largest velocity components towards the detector and thus small velocity components in directions perpendicular to the recoil ion beam axis.

\section{Conclusion.}

We have designed and tested at GANIL/SME a recoil ion source which can deliver very low energy beams of high charge-stage argon ions. The characteristics of these beams are such that their use in low energy charge exchange collision experiments may be immediately considered. These encouraging results are, for a large part, to be attributed to the excellent quality of the projectile ion beams available at GANIL/SME. Improvements which are now being brought to this accelerator will lead to increased intensities for the projectile beams and allow to anticipate even higher performances of our apparatus. 


\section{References}

[1] Cocke C. L., Olson R. E., Recoil ions, Phys. Rep. 205 (1991) 155-219.

[2] Tawara H., Phaneuf R. A., Atomic and Molecular Data Requirements for Fusion Plasma Edge Studies, Comments At. Mol. Phys. 21 (1988) 177-193.

[3] Justiniano E., Cocke C. L., Gray T. J., Dubois R. D.. Charge transfer and ionization in low-energy $\mathrm{Ar}^{\mathrm{q}+}+\mathrm{Ne}$ collisions, Phys. Rev. A 24 (1981) 2953-2962.

[4] Lembo L. J.. Danzmann K., Stoller Ch., Meyerhof W. E., Hänsch T. W., Core effects on the polarization of optical Rydberg transitions following electron capture into slow, highly ionized neon recoil ions, Phys. Rev. A 37 (1988) 1141-1151.

[5] Nielsen E. H., Andersen L. H., Barany A.. Cederquist H., Heinemeier J.. Hvelplund P., Knudsen H., Mc Adam K. B., Sorensen J., Energy-gain spectroscopy of state-selective electron capture for multiply charged Ar recoil lons, J. Phys. B. At Mol. Phys. 18 (1985) 1789-1808.

[6] Mc Cullough R. W., Wilson S. M. and Gilbody H. B., State-selective capture by slow $\mathrm{Ar}^{4+}, \mathrm{Ar}^{5+}$ and $\mathrm{Ar}^{6+}$ recoil ions in $\mathrm{H}, \mathrm{H}_{2}$ and He, J. Phys. B. At. Mol. Phys. 20 (1987) 2031-2055.

[7] Cederquist H., Biedermann C., Levin J. C., Sellin I. A., Short R. T., Production of highly-charged recoil-ion beams at very low energy, N.I.M.B 34 (1988) 243-251.

[8] Mann R., Total One-Electron Capture Cross Sections for $\mathrm{Ar}^{4+}$ and $\mathrm{I}^{9+}$ Ions in Slow Collisions on $\mathrm{H}_{2}$ and He, Z. Phys. D Atoms Molecular Cluster 3 (1986) 85-90.

[9] Mann R., Cocke C. L., Schlachter A. S., Prior M. and Marrus R., Selective Final-State Population in Electron Capture by Low-Energy Highly Charged Projectiles Studied by Energy-Gain Spectroscopy, Phys. Rev. Lett. 49 (1982) 1329-1332.

[10] Hennecart D.. Husson X., Lecler D., Lesteven-Vaïsse I., Grandin J. P., Multiple ionization of rare gases target atoms by $27 \mathrm{MeV} / \mathrm{amu} \mathrm{Xe}^{52+}$ and $35 \mathrm{Mev} / \mathrm{amu} \mathrm{Kr}^{36+}$ ions, Rad. Eff. Def. Solids 110 (1989) 141-143.

[11] Ulfrich J., Cocke C. L., Kelbch S., Mann R., Richard P., Schmidt-Böcking H., A parasite ion source for bare-ion production on a high-energy heavy-ion accelerator, J. Phys. B. At. Mol. Phys. 17 (1984) L 785-790.

[12] Malic R. A., Improved LEED system using position-sensitive detection, Rev. Sct. Instrum. 9 (1988) 1951-1953.

[13] Seliger R. L.. E $\times$ B Mass-Separator Design, J. Appl. Phys. 43 (1972) 2352-2357.

[14] Grandin J. P., Hennecart D., Husson X., Leclerc D., Lesteven-Vaïsse I., Lisfi D., Recoil Energy of Highly Charged Ions Produced in Swift Ion-Atom Collisions as Deduced from Time-of-Flight Measurements, Europhys. Lett. 6 (1988) 683-688.

[15] Ullrich J., Olson R., Schmıdt-Bockıng H., Schmidt S., Dörner R., Dangendorf V., Berg H., Multiple ionisation and collective electron emission in $\mathrm{MeV} / \mathrm{u}$ uranium-ion rare gas collisions, J. Phys. Colloq. France 50 (1989) C1-29-C1-36.

[16] Schlachter A. S., Groch W., Müller A., Beyer H. F., Mann R., Olson R. E., Production of highly charged rare-gas recoll ions by $1.4 \mathrm{MeV} / \mathrm{amu} \mathrm{U}^{44+}$ Phys. Rev. A 26 (1982) 1373-1377. 
